\title{
SIDRA: XML en la gestión y explotación de la documentación jurídica
}

\author{
Armando Alonso Peri \\ Consejería de Economía y Administración Pública \\ Gobierno del Principado de Asturias (España)
}

\section{Resumen}

En este artículo se explica la utilización del metalenguaje de marcado XML (eXtensible Markup Language) en el Sistema de Información Documental en Red de Asturias (SIDRA), haciendo especial referencia a la documentación legal del sistema, las Sentencias y el Boletín Oficial del Principado de Asturias (BOPA) como fuente de información jurídica. Se explica el proyecto SIDRA y la metodología empleada en la creación de una base de datos de Sentencias y del boletín electrónico (E-BOPA) dentro de dicho proyecto.

Palabras clave: XML. Sistema de Información Documental en Red de Asturias. SIDRA. Documentación jurídica. Bases de datos. Sistemas de información

\section{Abstract}

This paper explains the use of the XML markup language in the Asturias Online Document Information System (SIDRA), paying special attention to the system's legal documentation, the judgements, and the Asturias official gazette (BOPA) as source of legal information. The SIDRA project is also explained as well as the methodology used to create a judgements database and the online official gazette (E-BOPA)

Keywords: XML. Asturias Online Document Information System. SIDRA. Legal documentation. Databases. Information systems.

Podríamos definir el lenguaje XML (eXtensible Markup Language) como un metalenguaje de marcado que permite describir la estructura de tipos específicos de documentos electrónicos. Estas estructuras se definen por medio de DTD (Definición de Tipo de Documento) o XML Schemas (esquemas XML), mediante un conjunto de etiquetas $\mathrm{y}$ atributos.

La edición de documentos XML persigue los siguientes objetivos: $a$ ) distinguir el contenido y la estructura de los documentos de su presentación en papel o en pantalla; $b$ ) hacer explícita su estructura y sus contenidos informativos; $c$ ) crear 
documentos que puedan intercambiarse y procesarse con facilidad en sistemas informáticos heterogéneos.

Para alcanzar estos objetivos, XML propone un formato en el que se intercalan marcas en el texto de los documentos con el objeto de distinguir las partes o elementos estructurales del mismo.

Las posibilidades de intercambio de información entre sistemas informáticos heterogéneos que ofrece este lenguaje tienen un enorme potencial en el terreno de la documentación legal. Durante los últimos años han surgido en este ámbito iniciativas que persiguen el intercambio automático de información mediante la utilización de XML. Para ello es necesario crear estructuras de datos que permitan intercambiar información, pero alcanzar la estandarización es tarea difícil debido a la diversidad multicultural, sobre todo en Europa.

Las dos organizaciones pioneras en la estandarización de la información legal son LegalXML en Estados Unidos (sección de OASIS, Organisation for the Advancement of Structured Information Standards) (1) y LEXML en Europa (2). Las dos organizaciones persiguen la creación de un grupo de DTD para determinados tipos de documentos jurídicos.

LegalXML ha centrado sus esfuerzos en la intermediación entre operadores jurídicos norteamericanos con el fin de elaborar una DTD para todos los documentos de una clase determinada. Por su parte, la red LEXML se creó como foro para sustentar el interés creciente en el intercambio de datos legales. En este foro abierto se comparten ideas y experiencias asociadas a XML para el ámbito legal. Se trata de una red descentralizada pero que tiende a una aproximación paulatina a través de listas de distribución, asambleas y trabajo conjunto a través de proyectos internacionales. Este foro es el punto de coordinación y de trabajo para el desarrollo de estructuras estandarizadas, vocabularios y herramientas de intercambio de datos. Persigue un modelo de datos legal y global y el desarrollo de una fuente abierta de un programa oficial legal, que hable y entienda XML.

LEXML no acude a una estructura de datos por tipo de documento, como hace LegalXML; debido a la diversidad cultural, su objetivo es crear un buen número de estructuras de datos para las diferentes comunidades (Vañó y Broseta, 2005).

Estas iniciativas están consiguiendo importantes avances en la aplicación del lenguaje de marcado XML a la documentación jurídica.

Desde el Gobierno del Principado de Asturias se ha hecho un esfuerzo para la mejora del tratamiento específico este tipo de documentación. En este sentido se han llevado a cabo dos desarrollos: la base de datos de Sentencias para el Servicio Jurídico del Principado de Asturias y el Sistema para la Gestión y Edición Electrónica del Boletín Oficial del Principado de Asturias (E-BOPA). Se trata de dos iniciativas en las que se utiliza XML con documentación jurídica en el ámbito del

Scire. 15 : 1 (en.-jun. 2009) 111-124. ISSN 1135-3716. 
gobierno electrónico y que se enmarcan en el Sistema de Información Documental en Red de Asturias (SIDRA), del que hablaremos a continuación.

\section{El Sistema de Información Documental en Red de Asturias (SIDRA)}

En el año 2004 se creó el SIDRA para dar solución al inadecuado aprovechamiento de la información por parte del personal de la Administración del Principado de Asturias (gestores, directivos, administrativos...) y a la situación de los centros de documentación especializados, que no permitía ofrecer un servicio de calidad a los ciudadanos. Existía un claro aislamiento y una falta de coordinación entre los centros de documentación y las bibliotecas especializadas, y entre las unidades administrativas, sin la necesaria adecuación a las nuevas tecnologías para el cumplimiento de los objetivos marcados por el Gobierno asturiano, en concordancia con los mandatos españoles y europeos respecto al desarrollo de la Administración regional en el marco de la sociedad de la información.

El personal de la Administración lleva a cabo las más variadas funciones administrativas (jurídicas, técnicas, asistenciales, educativas, sanitarias, de asesoramiento, etcétera); sin embargo, se identificaron dos necesidades comunes a todos ellos: $a$ ) obtener en cada caso la información y el conocimiento precisos para el adecuado desempeño de sus funciones; $b$ ) tratar o procesar los documentos que soportan los trabajos realizados o las decisiones adoptadas, a efectos de la posible reutilización de la información en ellos recogida.

Para dar solución a la situación existente y atender estas necesidades se decidió crear, en el marco de las políticas de información, un sistema de información documental en red, siguiendo las líneas de actuación establecidas en el Plan de Modernización y Mejora de la Calidad en la Administración del Principado de Asturias. Fue entonces cuando la Dirección General de Modernización y Sistemas de Información (actualmente Dirección General de Modernización, Telecomunicaciones y Sociedad de la Información), a través del Servicio de Archivos Administrativos y Documentación (actualmente Servicio de Publicaciones, Archivos Administrativos y Documentación), puso en marcha el Sistema de Información Documental en Red de Asturias (SIDRA).

En estos momentos el SIDRA es un sistema integral de información documental en red ya consolidado. Está soportado por una única base de datos y en una única aplicación de gestión documental que permite el tratamiento, la recuperación, la distribución y, en su caso, el préstamo de todo tipo de documentación o información especializada, pública o restringida, necesaria para el ejercicio de las funciones de la Administración del Principado de Asturias. Se trata de un sistema dinámico que facilita el aprovechamiento de los recursos, el intercambio de información y la cooperación, con dos objetivos fundamentales: $a$ ) dar servicio a la sociedad asturiana, facilitando el acceso web a la información disponible en los

Scire. $15: 1$ (en.-jun. 2009) 111-124. ISSN 1135-3716. 
centros de documentación y bibliotecas especializadas de la Administración directa e institucional del Principado de Asturias; $b$ ) dar soporte a la organización del Principado de Asturias para la gestión y la toma de decisiones basadas en el conocimiento.

En la actualidad el SIDRA está integrado por los siguientes organismos: $a$ ) la Red de Centros de Documentación, compuesta actualmente por 21 unidades de información, entre las que se encuentran las del Ente Público de Servicios Tributarios del Principado de Asturias, el Consejo Económico y Social, la Oficina de Investigación Biosanitaria, la SADEI (Sociedad Asturiana de Estudios Económicos e Industriales), etcétera; $b$ ) el Servicio de Publicaciones, Archivos Administrativos y Documentación del Gobierno del Principado de Asturias, que es el coordinador del sistema y se ha encargado del desarrollo de los trabajos objeto de este artículo, la base de datos de Sentencias del Servicio Jurídico del Principado de Asturias y el Sistema para la Gestión y Edición Electrónica del Boletín Oficial del Principado de Asturias (E-BOPA); c) la Red Asturiana de Información Juvenil (RAIJ), compuesta por 39 Oficinas de Información Juvenil (OIJ); d) los gabinetes de las Consejerías del Gobierno del Principado; e) el Servicio Jurídico del Principado de Asturias.

\subsection{Software}

El SIDRA se ha desarrollado con tecnología estándar universal XML bajo la plataforma de Tamino y Dogma. Este software permite definir de forma abierta y flexible distintos modelos de datos y asociar a los registros todo tipo de documentos originales (archivos textuales, video, imagen, sonido...) tratados (captura, digitalización, indexación, versionados...) para garantizar su localización inmediata y la recuperación de su contenido. Los registros se almacenan en formato XML.

\subsubsection{Tamino}

Se trata de una base de datos centrada en documentos (data-centric database) que respeta la estructura de los mismos, permite hacer consultas sobre dicha estructura y hace posible la recuperación del documento tal como fue insertado originalmente. Destacamos algunas de sus características más importantes:

- Soporta información en diferentes formatos, debido a que les permite pasar su información a un formato XML rápidamente, utilizando la aplicación de su preferencia, y de esta manera almacenarla en una de estas bases de datos y evitarse el proceso de conversión de un formato a otro. Almacena casi cualquier tipo de documento incluyendo información formateada XML, páginas HTML, cartas, audio, vídeo, imágenes y datos de bases de datos relacionales o de objetos. Los documentos se agrupan en función de la información que contienen, en colecciones que a su vez pueden contener otras colecciones. 
- Provee tanto almacenamiento XML como mecanismos de almacenamiento relacional SQL. Esta característica permite a los usuarios consultar datos heterogéneos mediante XQL y recibir conjuntos de resultados en formato XML.

— Facilita el desarrollo de aplicaciones basadas en XML, como por ejemplo servicios web.

— Soporta dos lenguajes de consulta, el Tamino XQuery (basado en el estándar XPath) y el lenguaje de consulta estándar XQuery recomendado por el W3C.

— Indexación XML. Se ha de permitir la creación de índices que aceleren las consultas realizadas frecuentemente.

- Crea identificadores únicos. A cada documento XML se le asocia un identificador único por el que será reconocido dentro del repositorio.

\subsubsection{Dogma}

Se trata de la aplicación de gestión documental del SIDRA, soportada por Tamino. Dogma almacena de forma nativa los documentos en formato XML. El sistema se beneficia del uso completo y nativo de este estándar universal:

- La utilización de XML de forma nativa proporciona máxima rapidez en la respuesta a través de un navegador.

- XML está especialmente diseñado para mantener cualquier estructura de información por sencilla o compleja que esta sea.

- La estructura de la información puede ser totalmente libre o adaptarse a estándares universales, conviviendo las diversas opciones entre sí.

- Al tratarse de un estándar universal, XML facilita cualquier intercambio de información sin necesidad de conversiones.

- Transparencia en los datos: todo lo contrario que los lenguajes propietarios de los entornos tradicionales.

- Permite cualquier dispositivo de consulta: navegador, teléfonos móviles...

Dogma se basa en una arquitectura multiplataforma de tres capas organizadas de la siguiente forma:

- Datos. Los datos son almacenados en Tamino XML Server, servidor de información nativo XML capaz de almacenar y gestionar toda la documentación XML y no XML generada.

- Aplicación / framework. La aplicación Dogma está basada en el estándar J2EE, como IBM WebSher, BEA WebLogic, Macromedia Jrun, Apache Tomcat, etcétera.

- Presentación. A todas las funcionalidades de Dogma (incluidas las propias de administración de la aplicación) se accede a través de un navegador. 
Dogma es un sistema integrado que facilita y favorece el acceso a cualquier tipo de información. Incorpora una serie de funciones que permiten el mantenimiento de la información, así como su análisis y tratamiento. Sus funciones más importantes son localización, captura de información y mantenimiento, y administración. Otras características de Dogma son las siguientes:

- Centralización y categorización en un repositorio único.

- Máxima capacidad de respuesta a través de cualquier dispositivo de consulta. Permite la configuración de servicios web de acceso multicanal.

- Creación y mantenimiento de cualquier estructura de información por sencilla o compleja que sea, incluyendo tesauros o lenguajes controlados.

- Unificación de la comunicación mediante XML: sin necesidad de convertir entre formatos, la exportación se convierte en una utilidad del usuario.

- Dogma es un framework documental, lo que permite constituya una solución totalmente abierta y flexible, capaz de integrar cualquiera de las funciones documentales sin necesidad de alterar los procesos de funcionamiento.

- Contenidos dinámicos y personalizables.

- Funcionalidades que permiten crear sencillamente la propia estructura de la información, los puntos de acceso y las interfaces de usuario.

- Módulos parametrizables y escalables.

- Integración con el portal www.asturias.es, BD de Terceros, LDAP, CRM.

- Tratamiento documental: catalogación, indización...

- Captura automática y almacenamiento de documentos de texto, imagen, sonido y vídeo en diferentes formatos: .doc, .pdf, .jpg, .wma, .wmv, .mp3...

- Digitalización asistida por OCR.

- Campos controlados y ayudas configurables.

- Gestión de tesauros, clasificaciones y listas de materias.

- Soporte de flujo de trabajo (workflow).

- Versionado de documentos.

- Difusión selectiva de la información (DSI). Posibilidad de crear servicios de alerta que avisen a los usuarios de la aplicación, por ejemplo, sobre incorporaciones de fondos de un tema determinado a la base de datos.

- Circulación de documentos y gestión del préstamo.

- Definición de perfiles de usuarios y niveles de seguridad.

- Generación de informes y estadísticas a través de la herramienta XMLMART. Esta aplicación permite la explotación de la información almace- 
nada en el repositorio XML Tamino, mediante la elaboración de informes y estadísticas para la toma de decisiones.

El concepto de documento, para Dogma, es totalmente abierto, y agrupa desde descripciones (información de los atributos o metadatos) hasta múltiples objetos (imagen, vídeo, sonido, etcétera) para un mismo documento. El resultado es que el documento es visto como una agrupación simple o compleja de información. Dogma facilita de forma integrada el acceso a cualquier tipo de información e incorpora toda una serie de funciones que permiten el mantenimiento de la información, así como su análisis y tratamiento.

A continuación pasamos a explicar los dos proyectos objeto de este artículo que se han desarrollado con esta infraestructura tecnológica.

\section{Base de datos de Sentencias del Servicio Jurídico del Principado de Asturias}

La función de esta base de datos es la de permitir la recogida, tratamiento, recuperación y difusión de la jurisprudencia en la que son parte el Gobierno y la Administración del Principado de Asturias. No se han creado estructuras de datos tipo DTD o XML Schema para las sentencias, sino que se ha definido un modelo de datos sobre la infraestructura tecnológica preexistente en el SIDRA (Tamino y Dogma) para cargar los registros a los que se adjuntarán las sentencias a texto completo.

Los objetivos con los que se creó esta base de datos son los siguientes: a) dotar al Servicio Jurídico de un sistema de información, mediante su incorporación al SIDRA; $b$ ) mejorar el nivel de eficiencia, efectividad y satisfacción en el acceso a la información jurídica; $c$ ) facilitar el acceso inmediato a los activos documentales relevantes; $d$ ) generar nuevos servicios de valor añadido.

Partiendo de estos objetivos se realizó un análisis de las necesidades del Servicio Jurídico mediante una Auditoría de Información que se estructuró en seis fases: 1) análisis del fondo, requisitos especiales del servicio y necesidades; 2) identificación de las entidades documentales básicas; 3) definición de los atributos de las entidades documentales; 4) definición del nivel de seguridad de los documentos; 5) configuración de búsquedas; 6 ) análisis de los instrumentos de descripción (tesauros, clasificaciones, listas de materia...).

\subsection{Análisis del fondo, requisitos especiales del servicio y necesidades}

En colaboración con el Servicio Jurídico se detectaron unas necesidades específicas de tratamiento de documentos, flujos de trabajo e información estadística. Estas necesidades se concretaron en la decisión de crear una base de datos de Sentencias que permitiese recoger, tratar, recuperar y difundir la jurisprudencia en la que son parte el Gobierno y la Administración del Principado de Asturias. En el resto de esta sección explicamos con más detalle cada una de estas fases.

Scire. $15: 1$ (en.-jun. 2009) 111-124. ISSN 1135-3716. 


\subsection{Identificación de las entidades documentales básicas.} Análisis, tratamiento y propuesta de gestión normalizada

Se identificó un grupo homogéneo de documentación, las sentencias. El Servicio Jurídico proporcionó varios modelos de ellas, con una serie de elementos que se repiten en todas y cada una de ellas y que proporcionaron un marco sobre el que trabajar. Sobre estos datos se definió la siguiente propuesta: $a$ ) elaboración de una base de datos específica para el Servicio Jurídico, denominada Sentencias, compuesta por una entidad principal para esta clase de documentos; $b$ ) creación en dicha base de datos de una serie de entidades auxiliares que permitieran por un lado la normalización de los datos y por otra agilizar el proceso de carga de una serie de campos incluidos en la entidad principal.

Teniendo en cuenta que los nombres de los diferentes campos reflejan su contenido desde el punto de vista documental, no jurídico, y que pueden/deben ser modificados por el Servicio Jurídico, el esquema general de dichas entidades fue el siguiente:

Entidad principal: Sentencias

Entidades auxiliares:

1. Fallos de sentencias

2. Juzgado / tribunal

3. Recursos contra sentencia

4. Sentencias asuntos penales

5. Sentencias dictadas contra recursos contencioso-administrativos

\subsection{Definición de los atributos de las entidades documentales}

Para cada una de las nuevas entidades se definieron una serie de atributos (metadatos) específicos que se detallan a continuación.

\subsubsection{Entidad principal (Sentencias)}

Como entidad principal era la más compleja y la que contenía un mayor número de datos; además estaba relacionada con todas y cada una de las entidades auxiliares. El modelo de datos definido para esta entidad se fundamentó en la información proporcionada por el Servicio Jurídico del Principado de Asturias. Los atributos definidos son los siguientes:

Tipo de seguridad

Colectivo

Compartido con

Número de control

Datos de la sentencia

Número de autos

Número de expediente

Scire. $15: 1$ (en.-jun. 2009) 111-124. ISSN 1135-3716. 
Título de la sentencia

Juzgado/tribunal

Ponente

Jurisdicción

Tipo de procedimiento

Fechas

Fecha de la sentencia

Fecha de la sentencia en firme

Resultado del fallo de la sentencia

Asuntos contencioso-administrativos

Administración recurrida

Administración recurrente

Sentencias dictadas en los recursos

Asuntos laborales

Sentencias dictadas por los juzgados

Sentencias dictadas por las salas de lo social

Sentencias dictadas en recurso de suplicación. Administración recurrida

Sentencias dictadas en recurso de suplicación. Administración recurrente Asuntos penales

Asuntos penales

Asuntos civiles

Asuntos civiles

Recurso que cabe contra la sentencia

Tipo de recurso

Condena en costas

Clasificación

Descriptor

Notas

Nota pública

Nota interna

Documento anexo

Archivo

Localización

Centro

\subsubsection{Entidades auxiliares}

Se trata de entidades mucho más sencillas en cuanto a su definición. Estos campos controlados tienen valores preestablecidos y se han definido como entidades para facilitar su actualización y mantenimiento. Los atributos definidos para cada una de ellas fueron los siguientes:

Scire. $15: 1$ (en.-jun. 2009) 111-124. ISSN 1135-3716. 
Fallos de sentencias

Número de control, asignado por el sistema

Fallo: tipo de fallo de la sentencia

Descripción: comentario en texto libre sobre el fallo Juzgado/tribunal

Número de control, asignado por el sistema

Nombre del juzgado/tribunal

Código correspondiente a cada juzgado o tribunal

Recursos contra sentencia

Número de control, asignado por el sistema

Tipo de recurso

Descripción: comentario en texto libre sobre el fallo

Sentencias asuntos penales

Número de control, asignado por el sistema

Tipo de sentencia

Descripción: comentario en texto libre sobre el fallo

Sentencias dictadas contra recursos contencioso-administrativos

Número de control, asignado por el sistema

Tipo de sentencia

Descripción: comentario en texto libre sobre el fallo

\subsection{Definición del nivel de seguridad de los documentos}

SIDRA permite establecer los siguientes tipos de seguridad en documentos: $a$ ) información pública, a la que podrá acceder todo tipo de usuario a través de Internet; $b$ ) información interna, solo es accesible a través de Intranet; $c$ ) información restringida, a la que solo pueden acceder los usuarios que pertenezcan al Servicio Jurídico; $d$ ) información restringida compartida, que podrá estar disponible para otros colectivos o centros si así lo indican los responsables del Servicio Jurídico.

En el caso de las Sentencias, el nivel de seguridad que se definió fue el restringido, con la posibilidad de compartir en algún momento con otros colectivos si así lo indican los responsables del Servicio.

\subsection{Configuración de búsquedas}

De nuevo, para cada una de las entidades, principal y auxiliares, se definieron una serie de campos de búsqueda en función de las necesidades que se detectaron en el Servicio Jurídico y de un análisis documental de las mismas. Sobre cada uno de los atributos (metadatos) definidos para todas las entidades se pueden realizar tres tipos de búsquedas: textual, alfabética y jerárquica. Se configuró la búsqueda textual como criterio de búsqueda por defecto. En todas aquellas entidades que tengan definido más de un campo de búsqueda se pueden utilizar operadores lógicos $(y, o, n o)$ para combinar campos. A continuación explicamos cómo funcionan los tres tipos de búsqueda que permite realizar la aplicación. 


\subsubsection{Búsqueda alfabética}

Este método de búsqueda permite el acceso a una lista de documentos (de la misma naturaleza; leyes, informes, noticias, etcétera) ordenados por un criterio determinado (permite múltiples criterios de ordenación) y presenta los resultados en forma de extracto.

En este caso, la búsqueda guía intuitivamente a los usuarios por la información cuando estos no son conocedores de las técnicas de interrogación (operadores booleanos) o cuando se dirigen a un punto ya conocido.

\subsubsection{Búsqueda textual}

El usuario introduce un argumento de búsqueda y Dogma responde mostrando los documentos que satisfacen dicha demanda. El argumento de búsqueda se realiza sobre diversos puntos de acceso, que pueden ser formateados o textuales (texto completo). Los argumentos de búsqueda se pueden construir tanto mediante el uso de operadores booleanos $(y, o, n o)$, relacionales (mayor, menor...) o de proximidad (proximidad, entre...), así como también mediante el uso de truncamiento de caracteres (utilizando "asterisco" tanto a la derecha, como a la izquierda o en el medio de la cadena de caracteres). Los documentos resultantes de la búsqueda se mostrarán de forma extractada.

\subsubsection{Búsqueda jerárquica}

Este método de búsqueda permite acceder a los documentos que pertenecen al mismo grupo (leyes, circulares, noticias...) navegando por un árbol jerárquico. Es especialmente aconsejable para realizar búsquedas sobre tesauros y lenguajes controlados (como listas de materias).

\subsection{Análisis de los instrumentos de descripción (tesauros, clasificaciones, listas de materias...)}

El Servicio Jurídico del Principado de Asturias no utilizaba ningún instrumento de descripción normalizado. En la nueva base de datos de Sentencias se decidió incluir el tesauro Eurovoc, con el desarrollo propio del Senado (4). Esta decisión se tomó una vez analizadas otras posibles opciones (5).

Se trata de un tesauro muy completo y útil para el control de información general sobre diversos aspectos del ámbito socioeconómico y otros campos como el derecho, la educación o el medio ambiente.

\section{Sistema para la Gestión y Edición Electrónica del Boletín Oficial del Principado de Asturias (E-BOPA)}

El proyecto E-BOPA, que se encuentra actualmente en desarrollo, acomete la modernización de todos los procesos implicados en la publicación del Boletín 
Oficial del Principado de Asturias, desde el momento en que se produce la solicitud de publicación de un anuncio (6) hasta que esta publicación se lleva a efecto. También gestiona los productos de salida: el propio BOPA y su publicación en el portal corporativo, en el catálogo de servicios del portal, en la base de datos legislativa INVESDOC y en otros posibles servicios futuros al ciudadano, como un repositorio de instancias tipo para gestiones con la Administración del Principado o un servicio de difusión selectiva de la información (DSI) del BOPA.

Con este proyecto se pretende atender a las siguientes necesidades: gestión de los anuncios a publicar, edición de anuncios, montaje de boletines, mantenimiento de un histórico de anuncios y/o boletines, elaboración de informes, exportación a distintos formatos y recepción de anuncios multicanal.

Para conseguir estos objetivos se han creado dos aplicaciones basadas en la misma infraestructura tecnológica utilizada en el SIDRA (Tamino y Dogma): PUBLIBOPA (sistema de inserción digital de anuncios), aplicación que sirve de canal de entrada de los anuncios para su publicación en el BOPA, y SIGBOPA (Sistema para la Gestión y Publicación del Boletín Oficial del Principado de Asturias), aplicación en la que quedarán registrados por un lado los anuncios y por otro los boletines que se publiquen.

El esquema general sería el que sigue: las solicitudes de publicación se deben recoger de forma automatizada; la aplicación con su correspondiente BD se denominará PUBLIDOPA y tendrá dos vías diferenciadas de entrada: $a$ ) la propia Administración del Principado de Asturias; $b$ ) otras Administraciones: Administración Local, Administración de Justicia y Delegación de Gobierno.

Por otra parte, las solicitudes irán acompañadas del propio documento a publicar, que tiene que haber pasado por un proceso de autentificación/certificación que garantice al Servicio de Publicaciones, Archivos Administrativos y Documentación la autoría y responsabilidad legal del texto a publicar.

La aplicación PUBLIBOPA debe integrarse con SIGBOPA, y fruto de dicha integración se han de obtener los siguientes resultados:

- El registro de cada anuncio a publicar.

— El BOPA preparado para su impresión.

- Los modelos de solicitud preparados para su impresión.

A su vez, la aplicación SIGBOPA deberá integrarse con otras aplicaciones preexistentes con estos resultados:

— Integración con el portal www.asturias.es.

- BOPA en formato HTML publicado en el portal. Para la publicación en web, SIGBOPA remite un fichero XML. Se ha creado un XML Schema que define la estructura típica del BOPA.

Scire. $15: 1$ (en.-jun. 2009) 111-124. ISSN 1135-3716. 
- Anuncios en formato PDF publicados en el portal.

- Ficha del catálogo de servicios actualizada en el portal con modelo de solicitud. Se trata de una integración orientada a información que no requiere modificaciones en las aplicaciones integradas, sino solamente la implementación del mecanismo de transmisión de información entre los repositorios de datos de las aplicaciones respectivas.

— Integración con INVESDOC.

- Registro del anuncio en esta BD legislativa.

- Anuncio en formato PDF asociado a dicho registro.

\section{E-BOPA}

\section{Boletín Oficial del Principado de Asturias Electrónico}

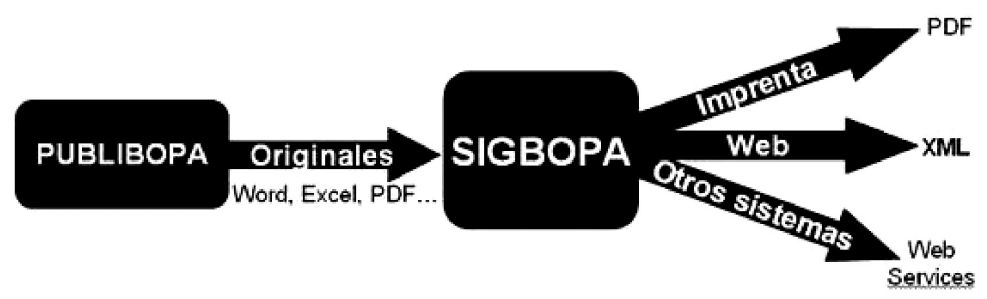

Figura 1. E-BOPA.

\section{Conclusiones}

La aplicación del lenguaje XML durante los últimos años en los procesos de gestión de contenidos ha significado un importante avance, tanto para el almacenamiento de los mismos y sus metadatos como para la integración y el intercambio de contenidos procedentes de aplicaciones o de organizaciones externas.

Las iniciativas desarrolladas desde la Administración del Principado de Asturias que hemos expuesto en este artículo pretenden conseguir precisamente esto, una mejora de los procesos de gestión de los contenidos que emanan de nuestra organización, de forma que ayuden al personal de la Administración en la toma de decisiones y en el desarrollo de sus tareas diarias. Todo ello va orientado a ofrecer mejores servicios a los ciudadanos.

En esta línea, desde nuestra Administración seguimos adaptándonos a estas nuevas tecnologías, que favorecen una adecuada gestión del conocimiento en la organización y un servicio de calidad. 


\section{Notas}

(1) LegalXML: http://www.legalxml.org/ (2008-03-26).

(2) LEXML-ES: http://www.uv.es/lexml/ (2008-03-26).

(3) Página del Catálogo de acceso en línea del Sistema de Información Documental en Red de Asturias: http://sidra.princast.es (2008-03-26).

(4) Acceso al tesauro Eurovoc: http://www.senado.es/basesdedatos/index.html (2008-03-26).

(5) Otros tesauros de interés: http://www.cindoc.csic.es (tesauro de derecho), http://www.westlaw.es (tesauro de Aranzadi; acceso restringido para usuarios registrados), http://www.bosch-online.net (tesauro específico de jurisprudencia) (2008-03-26).

(6) A efectos de este proyecto entenderemos por anuncio cada elemento de publicación en el BOPA.

\section{Referencias}

Alvite, María Luisa (2004). Evolución de las bases de datos jurídicas en España. // Anales de Documentación. 7 (2004) 7-27. http://www.um.es/fccd/anales/ad07/ad0701.pdf (2008-02-25).

Arellano, María del Carmen (2005). Aportaciones de la técnica legislativa y XML a la informática jurídica documental. Madrid: Universidad Carlos III, Boletín Oficial del Estado, 2005.

Bosch, Mela (2001). Documentos y lenguaje de marcado: conceptos, problemas y tendencias. // El profesional de la información. 10:11 (nov. 2001) 4-9.

Eíto, Ricardo (2005). XML y la gestión de contenidos = Content management and XML: the needed interaction. Hipertext.net.3 (mayo 2005). http://www.hipertext.net (200802-21).

Morrison, Michael (2000). XML al descubierto. Madrid: Prentice Hall, 2000.

Vañó, María José; Broseta, Manuel (2005). XML, una herramienta al servicio del buen gobierno corporativo. // Revista Aranzadi de Derecho y Nuevas Tecnologías. 8 (2005) 101-116.

Recibido: 2008-03-26. Aceptado: 2008-06-16

Scire. $15: 1$ (en.-jun. 2009) 111-124. ISSN 1135-3716. 